Original Research Article

\title{
Management of urinary tract infections in children: antimicrobial sensitivity pattern, efficacy and pharmacoeconomics
}

\author{
Nikhil S. Yadav*, Swanand S. Pathak
}

Department of Pharmacology, JNMC, Sawangi (Meghe), Wardha, Maharashtra, India

Received: 11 April 2019

Accepted: 07 May 2019

\section{*Correspondence to:} Dr. Nikhil S. Yadav, Email: mysticfugue@gmail.com

Copyright: (c) the author(s), publisher and licensee Medip Academy. This is an openaccess article distributed under the terms of the Creative Commons Attribution NonCommercial License, which permits unrestricted noncommercial use, distribution, and reproduction in any medium, provided the original work is properly cited.

\begin{abstract}
Background: Urinary tract infections are commonly seen in febrile children, high incidence of Vesico Ureteral Reflux (VUR) is frequently seen in paediatric patient which is of concern as it may lead to renal scarring. Despite presence of established clinical guidelines there is disparity amongst physician in the diagnosis and treatment of UTI, some physician prescribes taking into consideration the symptoms, some prescribe on the basis of smell and colour and some rely on urine culture and sensitivity report. There is dearth of studies in many tertiary health care centers regarding antimicrobial use. Authors conducted this study to evaluate antimicrobial sensitivity pattern, efficacy and cost effectiveness of antimicrobials used for UTI in children.

Methods: Patients of urinary tract infection $<13$ years of age were included in the study. Symptoms of patient i.e. pain in abdomen, haematuria, increased frequency of urination and degree of fever were recorded. Efficacy was measured through calculating degree of defervescence per hour. Cost effective model was prepared by calculating cost effective ratio i.e. dividing cost of antimicrobial required to bring down the fever and degree through which fever came down.

Results: E. coli was the most common pathogen isolated from urine positive culture $(69.07 \%)$. Nitrofurantoin has shown highest sensitivity to all uropathogens $(72.73 \%)$. Cotrimoxazole, ciprofloxacin and nitrofurantoin were found to be most cost effective.

Conclusions: In this current study authors found $E$ coli is the most common uropathogen isolated. Ceftriaxone, cefixime, cotrimoxazole and amikacin were found to be most efficacious. Cotrimoxazole, ciprofloxacin and nitrofurantoin were found to be most cost effective.
\end{abstract}

Keywords: Cost effective ratio, Fever, Urinary tract infection

\section{INTRODUCTION}

Urinary tract infection (UTI) of bacterial origin is seen frequently in children, infection of upper urinary tract (ureters and kidney) and lower urinary tract (bladder and urethra) or both, boys get frequently infected than girls during first year of life after which susceptibility in girls increases substantially. ${ }^{1,2}$ UTI accounts for 1.1 million physician visits annually, approximately $2 \%$ of boys and $8 \%$ of girls suffers from UTI in initial 8 years. ${ }^{3,4}$ Analysis of cumulative incidence of urinary tract infection in
American children indicates that around 180,000 children are likely to suffer from urinary tract infections till 6 years of age and around 12-30\% among these children will suffer from recurrent urinary tract infection. ${ }^{5}$

Urinary tract infection especially community acquired UTI are commonly seen in developing countries due to poor hygienic sanitation practices, UTI can be symptomatic or asymptomatic due to which the diagnosis of urinary tract infection is often missed. Young children often suffer from vague symptoms like irritability and fever, American 
academy of paediatrics (AAP), suggests that a young child with fever and without other symptom should be recognized as a potential case of urinary tract infection, Older children however presents with classic symptoms of urinary tract infection i.e. abdominal pain, burning micturition and fever. Children especially young children are more likely to suffer from long term complications of urinary tract infections like renal scarring and renal failure in adulthood. ${ }^{5}$

Urinary tract infections are commonly seen in febrile children, high incidence of Vesico Ureteral Reflux (VUR) is frequently seen in paediatric patient which is of concern as it may lead to renal scarring, recurrence of UTI etc., which further aggravates the problem. Fever is considered as a marker for urinary tract infection because it is recognized as marker of renal parenchymal involvement. ${ }^{6}$ Aggressive treatment of UTI is mandatory in children within 3 days of arrival in OPD to prevent renal damage (when culture sensitivity report is awaited), delay in treatment often leads to increased severity of infection with greater incidence of renal damage. ${ }^{7}$

Etiology and resistance pattern of urinary tract infection acquired through community is generally not available and if available is outdated as resistance pattern of bacteria to antimicrobials often changes periodically in a given region. The etiological agents responsible for causing UTI are different in different region and their variability changes with geographical location, time and age of the patients. Gram negative bacteria which are antimicrobial resistant are becoming a major concern because of few therapeutic options, Gram negative bacteria specifically Enterobacteriaceae are most common etiological agent causing urinary tract infection, these Enterobacteriaceae acquire genes which are responsible for resistance to various antimicrobials, the resistant organisms produces ESBL (extended spectrum beta lactamases), Amp-C beta lactamases etc. Escherichia coli, klebsiella pneumonia, enterococcus faecalis, staphylococcus aureus and pseudomonas accounts for majority of the cases of urinary tract infections in childrens. ${ }^{8,9}$

Recent reports of urinary tract infections caused by Enterobacteriaceae producing extended spectrum beta lactamases (ESBL) are seen in children. Emerging resistance of uropathogens to first generation cephalosporins, sulfamethoxazole/trimethoprim and fluroquinolones are causing researchers/physicians to search for alternatives based on culture sensitivity patterns of uropathogens in that community. Aminoglycosides, third generation cephalosporins and nitrofurantoin are emerging as a therapy for UTI with high sensitivity rates. ${ }^{10}$

The urinary tract infections are second most common infection in society accounting for huge economic burden on society, the antimicrobial sensitivity pattern of urinary tract infection within a specific community changes with time, empirical treatment of UTI leads to ineffective treatment and there is rise in antimicrobial resistance amongst pathogenic microorganism which further leads to increased economic burden on society, annual cost of community acquired infection is estimated to be 1.6 million US dollars. ${ }^{811}$ Cost effectiveness is a widely approached method to analyse whether the health benefits of a particular intervention is of considerable value relative to its costs so that its application within a particular arena is of value i.e. cost per unit health gained. Cost effective analysis therefore has immense utility in a developing country due to limited resources and more prevalent health problems. $^{12}$

Despite presence of established clinical guidelines there is disparity amongst physician in the diagnosis and treatment of UTI, some physician prescribe taking into consideration the symptoms alone, some prescribe on the basis of smell and colour and some rely on urine culture and sensitivity report. ${ }^{13}$ Patients of UTI are prone to recurrence and high costs of treatment leads to increase burden of costs of treatment on patients since UTI are a frequent cause of hospitalisation and have a great economic impact on healthcare systems. ${ }^{14}$ There is dearth of studies in many tertiary health care centers regarding antimicrobial use which leads to injudicious use of antimicrobials, which causes increased resistance and cost of treatment in society, community acquired UTI are one of the most common occurring infection treated empirically, so a periodic study to evaluate resistance pattern and prevalence of uropathogens are necessary to establish proper empirical therapy for that particular community. ${ }^{15}$ Therefore, authors had conducted this study.

The aims were to study the antimicrobial sensitivity patterns of urinary tract infections in children and comparing efficacy and cost effectiveness of prescribed antimicrobials.

The objectives were to study pattern of uropathogens in children suffering from urinary tract infection, to study antimicrobial sensitivity pattern of different uropathogens isolated from children suffering from urinary tract infection, to study and compare efficacy of different antimicrobials used in treatment of urinary tract infection in children and to study and compare cost effective ratio of different antimicrobials used in treatment of urinary tract infection in children.

\section{METHODS}

Present study was observational study carried out in the paediatrics ward and paediatric intensive care unit of Jawaharlal Nehru Medical College and Acharya Vinoba Bhave Rural Hospital, DMIMS, Sawangi (Meghe), Wardha, Maharashtra, India.

The study was initiated after approval of Institutional Ethics Committee (No. DMIMS(DU)/IEC/2016-17/4060).

This study was conducted in 2 years (September 2016 to September 2018). 
The sample size of patients of urinary tract infection were $<13$ years of age admitted in paediatric ward/paediatric intensive care unit for treatment were included in the study from September 2016 to September 2018, 100 such paediatric patients were selected for this study. Only patients who were diagnosed and suspected cases of urinary tract infection less than or equal to13 years of age were included in the study.

Written consent was taken from guardian/parent of the admitted patients through consent form.

\section{Inclusion criteria}

All diagnosed/suspected patients of UTI less than or equal to 13 years of age admitted in paediatric ward/PICU were included.

\section{Exclusion criteria}

\section{OPD patients}

Patients with complicated urinary tract infection (i.e. functional or structural abnormality of urinary tract).

\section{Critically ill patients}

Patients whose parents/guardians are not willing to involve their ward in study.

\section{Procedure}

Symptoms of patient i.e. pain in abdomen, haematuria, increased frequency of urination and degree of fever were recorded, fever of patient were recorded using axillary temperature via thermometer, temperature $>37^{\circ} \mathrm{C}$ was considered as fever.

Midstream urine was collected under strict aseptic condition, $3 \mathrm{ml}$ of urine is collected in a bulb and sent to microbiology lab. On agar plate urine is placed and incubated at a temperature equal to normal human temperature, any uropathogen if present will grow in circular colonies in 48 hours, growth $>105 \mathrm{CFU} / \mathrm{ml}$ was considered significant.

Efficacy of antimicrobials was compared by measuring the fever of patients on arrival, patients fever pattern were followed through TPR (temperature, pulse and respiration) chart 4 hourly and time required for fever to come down was noted in hours, time required for defervescence (the abatement of fever as indicated by a decrease in bodily temperature) was noted for different antimicrobials and compared.

Efficacy was also be measured through calculating degree of defervescence per hour, degree of defervescence/hour for different antibiotics was be noted and compared. Cost effective model was prepared by calculating cost effective ratio i.e. dividing cost of antimicrobial required to bring down the fever and degree through which fever came down.

Cost Effective ratio $=$ Cost of antimicrobial required to bring down the fever/Degree through which fever came down.

Statistical analysis was done using chi square test and multiple comparison tukey test and software used in the analysis was SPSS 22.0 and $\mathrm{p}<0.05$ was considered significant.

\section{RESULTS}

The present study was conducted to investigate the antimicrobial sensitivity pattern, bacteriological profile and compare efficacy and cost-effective analysis of antimicrobials used in children suffering from urinary tract infection.

Table 1: Age wise distribution of patients.

\begin{tabular}{|ll|l|}
\hline Age group (yrs) & No. of patients & Percentage (\%) \\
\hline $1-4$ yrs & 43 & 43 \\
\hline $5-8$ yrs & 29 & 29 \\
\hline $9-13$ yrs & 28 & 28 \\
\hline Total & 100 & 100 \\
\hline Mean \pm SD & $5.89 \pm 3.65(1-13$ yrs $)$ \\
\hline
\end{tabular}

Table 1 showing age wise distribution of children suffering from UTI, $43 \%$ of children comes in age group of 1-4 years, $29 \%$ of children comes in age group of 5-8 years, $28 \%$ of children comes in age group of 9-13 years. The mean age of children was $5.89 \pm 3.65$ years.

Table 2: Gender wise distribution of patients suffering from UTI.

\begin{tabular}{|ll|l|}
\hline Gender & No. of patients & Percentage (\%) \\
\hline Male & 54 & 54 \\
\hline Female & 46 & 46 \\
\hline Total & 100 & 100 \\
\hline
\end{tabular}

Table 2 shows gender wise distribution of patients suffering from UTI. 54\% of patients are male and remaining $46 \%$ of patients are female.

Table 3: Distribution of patients based on urine culture report.

\begin{tabular}{|lll|}
\hline Culture report & No. of patients & Percentage \\
\hline Culture positive & 55 & 55 \\
\hline Culture negative & 45 & 45 \\
\hline Total & 100 & 100 \\
\hline
\end{tabular}

Table 3 shows distribution of patients based on urine culture report. $55 \%$ of all urine culture reports are culture positive and $45 \%$ are culture negative. 
Table 4: Gender wise distribution of growth of organism.

\begin{tabular}{|llll|}
\hline Growth of organism & Male (54) & Female (46) & Total \\
\hline Culture positive & 27 & 28 & 55 \\
\hline Percentage & $49.09 \%$ & $50.91 \%$ & $100 \%$ \\
\hline
\end{tabular}

Table 4 shows gender wise distribution of growth of organisms on urine culture. $50.91 \%$ of culture positive cases of UTI were females and $49.09 \%$ were males.

Table 5: Distribution of patients according to symptoms.

\begin{tabular}{|lll|}
\hline Symptoms & $\begin{array}{l}\text { No. of } \\
\text { patients }\end{array}$ & $\%$ \\
\hline Fever & 100 & 100 \\
\hline Abdominal pain & 46 & 46 \\
\hline Burning micturation & 37 & 37 \\
\hline Increased frequency of micturition & 24 & 24 \\
\hline Haematuria & 10 & 10 \\
\hline
\end{tabular}

Table 5 showing distribution of patients suffering from UTI according to symptoms. All (100\%) patients admitted in paediatric wards were suffering from fever out of which $46 \%$ of patients were having abdominal pain, $37 \%$ of patients were having burning micturition, $24 \%$ of patients were having increased frequency of urination and $10 \%$ of patients were having haematuria.

Table 6: Distribution of patients according to antimicrobials used.

\begin{tabular}{|lll|}
\hline Antibiotics used & No. of patients & $\%$ \\
\hline Inj Amikacin & 13 & 13 \\
\hline Inj Cefotaxime & 1 & 1 \\
\hline Inj Ceftriaxone & 21 & 21 \\
\hline Inj Imipenem & 1 & 1 \\
\hline Syp/Tablet Cefixime & 16 & 16 \\
\hline Syp/Tablet Cefopodoxime & 3 & 3 \\
\hline Syp/Tablet Coamoxyclav & 7 & 7 \\
\hline Syp Cotrimoxazole & 9 & 9 \\
\hline Tab Ciprofloxacin & 9 & 9 \\
\hline Tab Nitrofurantoin & 20 & 20 \\
\hline Total & 100 & 100 \\
\hline
\end{tabular}

Table 6 showing distribution of patients according to antimicrobials used, in $21 \%$ patients of UTI Inj. ceftriaxone was used, Tab nitrofurantoin was used in $20 \%$ of patients, Syrup/Tablet cefixime was used in $16 \%$ of patients, Inj. amikacin was used in $13 \%$ of patients, Syrup cotrimoxazole and Tablet ciprofloxacin were used in $9 \%$ of patients.

Syrup/Tablet comoxyclav and cefopodoxime were used in $7 \%$ and $3 \%$ of patients respectively, Inj. cefotaxime and imipenem were used in $1 \%$ of patients respectively.
Table 7: Distribution of patients according to temperature on arrival.

\begin{tabular}{|llc|}
\hline Temperature on arrival & No. of patients & $\%$ \\
\hline $38.1-38.5$ & 14 & 14 \\
\hline $38.6-39$ & 54 & 54 \\
\hline $39.1-39.5$ & 16 & 16 \\
\hline $39.6-40$ & 16 & 16 \\
\hline Total & 100 & 100 \\
\hline Mean \pm SD & $39.07 \pm 0.48$ & $(39.10-40)$ \\
\hline
\end{tabular}

Table 7 shows mean temperature of UTI patients on arrival i.e. $39.07 \pm 0.48(39.10-40)^{\circ} \mathrm{C}$. Distribution of UTI patients based on body temperature on arrival. $14 \%$ of UTI patients were having temperature in range of 38.1-38.5 degree Celsius. $54 \%$ of patients were having temperature in range of 38.6-39 degree Celsius, $16 \%$ of patients were having temperature in range of 39.1-39.5 degree Celsius and remaining $16 \%$ of patients were having fever in range of 39.6-40 degree Celsius.

Table 8: Distribution of patients according to growth of organism.

\begin{tabular}{|lll|}
\hline Growth of organism & No. of patients & $\%$ \\
\hline Acinetobacter spp & 2 & 3.64 \\
\hline E. coli & 38 & 69.09 \\
\hline Enterococcus faecalis & 2 & 3.64 \\
\hline Klebsiella pneumoniae & 10 & 18.18 \\
\hline Pseudomonas & 1 & 1.82 \\
\hline Staphylococcus aureus & 2 & 3.64 \\
\hline
\end{tabular}

Table 8 showing distribution of patients according to growth organism. The predominant isolate was E. coli (69.09\%) followed by Klebsiella pneumonia (18.18\%), other uropathogens isolated are Acinetobacter spp (3.64\%), Enterococcus faecalis (3.64\%), Staphylococcus aureus (3.64\%) and Pseudomonas (1.82\%) respectively.

Authors did post Hoc Tukey's test to compare mean time required for defervescence for different antimicrobials used in UTI, no significant difference was found in between mean time required for defervescence of all antibiotics except nitrofurantoin ( $\mathrm{p}$-value $>0.05$ ), when mean time required for defervescence was compared between all other antimicrobials (ceftriaxone, cefixime, cefpodoxime, ciprofloxacin, cotrimoxazole, amikacin and coamoxyclav) and nitrofurantoin, significant difference was found in mean time required for defervescence ( $p$ value <0.05). Authors did post Hoc Tukey's test to compare mean defervescence/hour for different antimicrobials used in UTI, significant difference in mean defervescence/hour was found between amikacin and nitrofurantoin, ceftriaxone and (ciprofloxacin, ceftriaxone and coamoxyclav), ceftriaxone and nitrofurantoin, cefixime and nitrofurantoin, cotrimoxazole and nitrofurantoin ( $\mathrm{p}$-value $<0.05$ ). Imipenem and cefotaxime were not taken into consideration for statistical analysis since only one patient received each of this antimicrobial. 
Table 9: Multiple comparison of mean time required (in hours) for defervescence for different anti-microbials using Post Hoc Tukey test.

\begin{tabular}{|c|c|c|c|c|c|c|}
\hline \multirow{2}{*}{ Antimicrobials } & & \multirow{2}{*}{$\begin{array}{l}\text { Mean Diff. } \\
\text { (I-J) }\end{array}$} & \multirow{2}{*}{$\begin{array}{l}\text { Std. } \\
\text { Error }\end{array}$} & \multirow{2}{*}{ P value } & \multicolumn{2}{|c|}{ 95\% Confidence Interval } \\
\hline & & & & & Lower bound & Upper bound \\
\hline \multirow{7}{*}{ Inj Amikacin } & Inj Ceftriaxone & 2.67399 & 1.96437 & $0.872, \mathrm{NS}$ & -3.4222 & 8.7702 \\
\hline & Syp/Tab Cefixime & -0.10577 & 2.07842 & $1.000, \mathrm{NS}$ & -6.5559 & 6.3444 \\
\hline & Syp/Tab Cefopodoxime & -3.56410 & 3.56527 & $0.973, \mathrm{NS}$ & -14.6286 & 7.5004 \\
\hline & Syp/Tab Coamoxyclav & -4.23077 & 2.60951 & $0.736, \mathrm{NS}$ & -12.3292 & 3.8676 \\
\hline & Syp Cotrimoxazole & 0.65812 & 2.41370 & $1.000, \mathrm{NS}$ & -6.8326 & 8.1488 \\
\hline & Tab Ciprofloxacin & -3.34188 & 2.41370 & $0.862, \mathrm{NS}$ & -10.8326 & 4.1488 \\
\hline & Tab Nitrofurantoin & $-19.93077^{*}$ & 1.98306 & $0.0001, \mathrm{~S}$ & -26.0850 & -13.7765 \\
\hline \multirow{6}{*}{ Inj ceftriaxone } & Syp/Tab Cefixime & -2.77976 & 1.84712 & $0.803, \mathrm{NS}$ & -8.5121 & 2.9526 \\
\hline & Syp/Tab Cefopodoxime & -6.23810 & 3.43558 & $0.611, \mathrm{NS}$ & -16.9001 & 4.4239 \\
\hline & Syp/Tab Coamoxyclav & -6.90476 & 2.42932 & $0.098, \mathrm{NS}$ & -14.4439 & 0.6344 \\
\hline & Syp Cotrimoxazole & -2.01587 & 2.21766 & $0.984, \mathrm{NS}$ & -8.8982 & 4.8664 \\
\hline & Tab Ciprofloxacin & -6.01587 & 2.21766 & $0.132, \mathrm{NS}$ & -12.8982 & 0.8664 \\
\hline & Tab Nitrofurantoin & $-22.60476^{*}$ & 1.73913 & $0.0001, \mathrm{~S}$ & -28.0020 & -17.2075 \\
\hline \multirow{5}{*}{$\begin{array}{l}\text { Syp/Tablet } \\
\text { Cefixime }\end{array}$} & Syp/Tab Cefopodoxime & -3.45833 & 3.50204 & $0.975, \mathrm{NS}$ & -14.3266 & 7.4099 \\
\hline & Syp/Tab Coamoxyclav & -4.12500 & 2.52243 & $0.728, \mathrm{NS}$ & -11.9531 & 3.7031 \\
\hline & Syp Cotrimoxazole & 0.76389 & 2.31928 & $1.000, \mathrm{NS}$ & -6.4338 & 7.9616 \\
\hline & Tab Ciprofloxacin & -3.23611 & 2.31928 & $0.857, \mathrm{NS}$ & -10.4338 & 3.9616 \\
\hline & Tab Nitrofurantoin & $-19.82500^{*}$ & 1.86699 & $0.0001, \mathrm{~S}$ & -25.6190 & -14.0310 \\
\hline \multirow{4}{*}{$\begin{array}{l}\text { Syp/Tablet } \\
\text { Cefopodoxime }\end{array}$} & Syp/Tab Coamoxyclav & -0.66667 & 3.84110 & $1.000, \mathrm{NS}$ & -12.5872 & 11.2538 \\
\hline & Syp Cotrimoxazole & 4.22222 & 3.71085 & $0.947, \mathrm{NS}$ & -7.2941 & 15.7385 \\
\hline & Tab Ciprofloxacin & 0.22222 & 3.71085 & $1.000, \mathrm{NS}$ & -11.2941 & 11.7385 \\
\hline & Tab Nitrofurantoin & $-16.36667^{*}$ & 3.44630 & $0.0001, \mathrm{~S}$ & -27.0619 & -5.6714 \\
\hline \multirow{3}{*}{$\begin{array}{l}\text { Syp/Tablet } \\
\text { Coamoxyclav }\end{array}$} & Syp Cotrimoxazole & 4.88889 & 2.80514 & $0.659, \mathrm{NS}$ & -3.8166 & 13.5944 \\
\hline & Tab Ciprofloxacin & 0.88889 & 2.80514 & $1.000, \mathrm{NS}$ & -7.8166 & 9.5944 \\
\hline & Tab Nitrofurantoin & $-15.70000^{*}$ & 2.44446 & $0.0001, \mathrm{~S}$ & -23.2862 & -8.1138 \\
\hline \multirow{2}{*}{ Syp Cotrimoxazole } & Tab Ciprofloxacin & -4.00000 & 2.62397 & $0.792, \mathrm{NS}$ & -12.1432 & 4.1432 \\
\hline & Tab Nitrofurantoin & $-20.58889^{*}$ & 2.23423 & $0.0001, \mathrm{~S}$ & -27.5226 & -13.6552 \\
\hline Tab Ciprofloxacin & Tab Nitrofurantoin & $-16.58889^{*}$ & 2.23423 & $0.0001, \mathrm{~S}$ & -23.5226 & -9.6552 \\
\hline
\end{tabular}

Authors did post Hoc Tukey test to compare mean costeffective ratio (cost of antimicrobial in rupees/degree of defervescence) of different antimicrobial used in UTI.

Significant difference in mean cost-effective ratio (cost of antimicrobial in rupees/degree of defervescence) was found in between amikacin and all other anti-microbials (except ceftriaxone and coamoxyclav) (p-value <0.05).

Significant difference in mean cost-effective ratio (cost of antimicrobial in rupees/degree of defervescence) was also found in between ceftriaxone and other antimicrobials i.e. cefpodoxime, cefixime, cotrimoxazole, ciprofloxacin and nitrofurantoin (except coamoxyclav) (p-value <0.05).

Significant difference in mean cost-effective ratio (cost of antimicrobial in rupees/degree of defervescence) was found in between cefixime and coamoxyclav ( $\mathrm{p}$-value $<0.05)$. Significant difference in mean cost-effective ratio (cost of antimicrobial in rupees/degree of defervescence) was found in between coamoxyclav and (ciprofloxacin, cotrimoxazole and nitrofurantoin) (p-value $<0.05)$.

Imipenem and cefotaxime were not taken into consideration for statistical analysis since only one patient received each of this antimicrobial.

All the uropathogens showed highest sensitivity to nitrofurantoin $(72.73 \%)(2 \aleph$-value $=68.31$, p-value $=0.044$, Significant) followed by amikacin (29.09\%), cefixime $(27.27 \%)$, ceftriaxone $(23.64 \%)$, imipenem $(23.64 \%)$, ciprofloxacin (14.55\%), cotrimoxazole $(9.09 \%)$ cefpodoxime $(7.27 \%)$, coamoxyclav $(1.82 \%)$, ceftazidime $(1.82 \%)$ and cefotaxime $(1.82 \%)$ respectively. 
Table 10: Multiple comparison of degree of defervescence/hour for different antimicrobials using Post Hoc Tukeys test.

\begin{tabular}{|c|c|c|c|c|c|c|}
\hline \multirow{2}{*}{ Antimicrobials } & & \multirow{2}{*}{$\begin{array}{l}\text { Mean Diff. } \\
\text { (I-J) }\end{array}$} & \multirow{2}{*}{ Std. Error } & \multirow{2}{*}{ P value } & \multicolumn{2}{|c|}{ 95\% Confidence Interval } \\
\hline & & & & & Lower bound & Upper bound \\
\hline \multirow{7}{*}{ Inj Amikacin } & Inj. Ceftriaxone & -0.00737 & 0.00506 & $0.829, \mathrm{NS}$ & -0.0231 & 0.0083 \\
\hline & Syp/Tab Cefixime & 0.00354 & 0.00536 & $0.998, \mathrm{NS}$ & -0.0131 & 0.0202 \\
\hline & Syp/Tab Cefopodoxime & 0.00821 & 0.00919 & $0.986, \mathrm{NS}$ & -0.0203 & 0.0367 \\
\hline & Syp/Tab Coamoxyclav & 0.01340 & 0.00673 & $0.493, \mathrm{NS}$ & -0.0075 & 0.0343 \\
\hline & Syp Cotrimoxazole & -0.00157 & 0.00622 & $1.000, \mathrm{NS}$ & -0.0209 & 0.0177 \\
\hline & Tab Ciprofloxacin & 0.01298 & 0.00622 & $0.431, \mathrm{NS}$ & -0.0063 & 0.0323 \\
\hline & Tab Nitrofurantoin & $0.02719^{*}$ & 0.00511 & $0.0001, \mathrm{~S}$ & 0.0113 & 0.0431 \\
\hline \multirow{6}{*}{ Inj ceftriaxone } & Syp/Tab Cefixime & 0.01090 & 0.00476 & $0.311, \mathrm{NS}$ & -0.0039 & 0.0257 \\
\hline & Syp/Tab Cefopodoxime & 0.01557 & 0.00886 & $0.649, \mathrm{NS}$ & -0.0119 & 0.0431 \\
\hline & Syp/Tab Coamoxyclav & $0.02076^{*}$ & 0.00626 & $0.028, \mathrm{~S}$ & 0.0013 & 0.0402 \\
\hline & Syp Cotrimoxazole & 0.00579 & 0.00572 & $0.971, \mathrm{NS}$ & -0.0119 & 0.0235 \\
\hline & Tab Ciprofloxacin & $0.02035^{*}$ & 0.00572 & $0.013, \mathrm{~S}$ & 0.0026 & 0.0381 \\
\hline & Tab Nitrofurantoin & $0.03455^{*}$ & 0.00448 & $0.0001, \mathrm{~S}$ & 0.0206 & 0.0485 \\
\hline \multirow{5}{*}{ Syp/Tab Cefixime } & Syp/Tab Cefopodoxime & 0.00467 & 0.00903 & $1.000, \mathrm{NS}$ & -0.0233 & 0.0327 \\
\hline & Syp/Tab Coamoxyclav & 0.00986 & 0.00650 & $0.797, \mathrm{NS}$ & -0.0103 & 0.0300 \\
\hline & Syp Cotrimoxazole & -0.00511 & 0.00598 & $0.989, \mathrm{NS}$ & -0.0237 & 0.0134 \\
\hline & Tab Ciprofloxacin & 0.00944 & 0.00598 & $0.761, \mathrm{NS}$ & -0.0091 & 0.0280 \\
\hline & Tab Nitrofurantoin & $0.02365^{*}$ & 0.00481 & $0.0001, \mathrm{~S}$ & 0.0087 & 0.0386 \\
\hline \multirow{4}{*}{$\begin{array}{l}\text { Syp/Tab } \\
\text { Cefopodoxime }\end{array}$} & Syp/Tab Coamoxyclav & 0.00519 & 0.00990 & $1.000, \mathrm{NS}$ & -0.0255 & 0.0359 \\
\hline & Syp Cotrimoxazole & -0.00978 & 0.00957 & $0.970, \mathrm{NS}$ & -0.0395 & 0.0199 \\
\hline & Tab Ciprofloxacin & 0.00478 & 0.00957 & $1.000, \mathrm{NS}$ & -0.0249 & 0.0345 \\
\hline & Tab Nitrofurantoin & 0.01898 & 0.00888 & $0.400, \mathrm{NS}$ & -0.0086 & 0.0466 \\
\hline \multirow{3}{*}{$\begin{array}{l}\text { Syp/Tab } \\
\text { Coamoxyclav }\end{array}$} & Syp Cotrimoxazole & -0.01497 & 0.00723 & $0.442, \mathrm{NS}$ & -0.0374 & 0.0075 \\
\hline & Tab Ciprofloxacin & -0.00041 & 0.00723 & $1.000, \mathrm{NS}$ & -0.0229 & 0.0220 \\
\hline & Tab Nitrofurantoin & 0.01379 & 0.00630 & $0.368, \mathrm{NS}$ & -0.0058 & 0.0333 \\
\hline \multirow{2}{*}{ Syp Cotrimoxazole } & Tab Ciprofloxacin & 0.01456 & 0.00676 & $0.391, \mathrm{NS}$ & -0.0064 & 0.0355 \\
\hline & Tab Nitrofurantoin & $0.02876^{*}$ & 0.00576 & $0.0001, \mathrm{~S}$ & 0.0109 & 0.0466 \\
\hline Tab Ciprofloxacin & Tab Nitrofurantoin & 0.01421 & 0.00576 & $0.223, \mathrm{NS}$ & -0.0037 & 0.0321 \\
\hline
\end{tabular}

Table 11: Multiple comparison of cost-effective ratio (cost of antimicrobials in rupees/degree of defervescence) of antimicrobials used in UTI using Post Hoc Tukey test.

\begin{tabular}{|c|c|c|c|c|c|c|}
\hline \multirow{2}{*}{ Antimicrobials } & & \multirow{2}{*}{$\begin{array}{l}\text { Mean Diff. } \\
\text { (I-J) }\end{array}$} & \multirow{2}{*}{$\begin{array}{l}\text { Std. } \\
\text { Error }\end{array}$} & \multirow{2}{*}{$P$ value } & \multicolumn{2}{|c|}{ 95\% Confidence Interval } \\
\hline & & & & & Lower bound & Upper bound \\
\hline \multirow{7}{*}{ Inj Amikacin } & Inj Ceftriaxone & -5.11 & 3.52 & $0.831, \mathrm{NS}$ & -16.06 & 5.83 \\
\hline & Syp/Tab Cefixime & 24.90 & 3.73 & $0.0001, \mathrm{~S}$ & 13.31 & 36.48 \\
\hline & Syp/Tab Cefopodoxime & 21.80 & 6.40 & $0.021, \mathrm{~S}$ & 1.93 & 41.68 \\
\hline & Syp/Tab Coamoxyclav & 4.92 & 4.68 & $0.965, \mathrm{NS}$ & -9.62 & 19.47 \\
\hline & Syp Cotrimoxazole & 30.93 & 4.33 & $0.0001, \mathrm{~S}$ & 17.48 & 44.39 \\
\hline & Tab Ciprofloxacin & 25.38 & 4.33 & $0.0001, \mathrm{~S}$ & 11.92 & 38.83 \\
\hline & Tab Nitrofurantoin & 21.93 & 3.56 & $0.0001, \mathrm{~S}$ & 10.878 & 32.98 \\
\hline \multirow{6}{*}{ Inj Ceftriaxone } & Syp/Tab Cefixime & 30.02 & 3.31 & $0.0001, \mathrm{~S}$ & 19.72 & 40.31 \\
\hline & Syp/Tab Cefopodoxime & 26.92 & 6.17 & $0.001, \mathrm{~S}$ & 7.77 & 46.07 \\
\hline & Syp/Tab Coamoxyclav & 10.04 & 4.36 & $0.304, \mathrm{NS}$ & -3.49 & 23.58 \\
\hline & Syp Cotrimoxazole & 36.05 & 3.98 & $0.0001, \mathrm{~S}$ & 23.69 & 48.41 \\
\hline & Tab Ciprofloxacin & 30.50 & 3.98 & $0.0001, \mathrm{~S}$ & 18.13 & 42.86 \\
\hline & Tab Nitrofurantoin & 27.05 & 3.12 & $0.0001, \mathrm{~S}$ & 17.35 & 36.74 \\
\hline \multirow{5}{*}{ Syp/Tab Cefixime } & Syp/Tablet cefopodoxime & -3.09 & 6.29 & $1.000, \mathrm{NS}$ & -22.62 & 16.42 \\
\hline & Syp/TabletCoamoxyclav & -19.97 & 4.53 & $0.001, \mathrm{~S}$ & -34.04 & -5.91 \\
\hline & Syp Cotrimoxazole & 6.03 & 4.16 & $0.832, \mathrm{NS}$ & -6.89 & 18.96 \\
\hline & Tab Ciprofloxacin & 0.47 & 4.16 & $1.000, \mathrm{NS}$ & -12.44 & 13.40 \\
\hline & Tab Nitrofurantoin & -2.97 & 3.35 & $0.987, \mathrm{NS}$ & -13.37 & 7.43 \\
\hline \multirow{4}{*}{ Syp/Tab Cefopodoxime } & Syp/TabletCoamoxyclav & -16.88 & 6.89 & $0.232, \mathrm{NS}$ & -38.29 & 4.53 \\
\hline & Syp Cotrimoxazole & 9.13 & 6.66 & $0.868, \mathrm{NS}$ & -11.55 & 29.81 \\
\hline & Tab Ciprofloxacin & 3.57 & 6.66 & $0.999, \mathrm{NS}$ & -17.10 & 24.26 \\
\hline & Tab Nitrofurantoin & 0.12 & 6.19 & $1.000, \mathrm{NS}$ & -19.08 & 19.33 \\
\hline \multirow{3}{*}{ Syp/Tab Coamoxyclav } & Syp Cotrimoxazole & 26.01 & 5.03 & $0.0001, \mathrm{~S}$ & 10.37 & 41.65 \\
\hline & Tab Ciprofloxacin & 20.45 & 5.03 & $0.003, \mathrm{~S}$ & 4.82 & 36.09 \\
\hline & Tab Nitrofurantoin & 17.00 & 4.39 & $0.005, \mathrm{~S}$ & 3.38 & 30.63 \\
\hline \multirow{2}{*}{ Syp Cotrimoxazole } & Tab Ciprofloxacin & -5.55 & 4.71 & $0.936, \mathrm{NS}$ & -20.18 & 9.07 \\
\hline & Tab Nitrofurantoin & -9.00 & 4.01 & $0.336, \mathrm{NS}$ & -21.46 & 3.44 \\
\hline Tab Ciprofloxacin & Tab Nitrofurantoin & -3.44 & 4.01 & 0.989, NS & -15.90 & 9.00 \\
\hline
\end{tabular}


Table 12: Correlation between growth of some major organisms isolated and culture sensitivity.

\begin{tabular}{|lll|lllllll|}
\hline $\begin{array}{l}\text { Growth of } \\
\text { organism }\end{array}$ & Amikacin & Cefixime & $\begin{array}{l}\text { Cefta- } \\
\text { zidime }\end{array}$ & $\begin{array}{l}\text { Ceftria- } \\
\text { xone }\end{array}$ & $\begin{array}{l}\text { Cipro- } \\
\text { floxacin }\end{array}$ & $\begin{array}{l}\text { Coam- } \\
\text { oxyclav }\end{array}$ & $\begin{array}{l}\text { Imi- } \\
\text { penem }\end{array}$ & $\begin{array}{l}\text { Nitro- } \\
\text { furantoin }\end{array}$ & $\begin{array}{l}\text { Cef- } \\
\text { podoxime }\end{array}$ \\
\hline $\begin{array}{l}\text { Acineto- } \\
\text { bacter } \text { spp }\end{array}$ & $0(0 \%)$ & $0(0 \%)$ & $0(0 \%)$ & $0(0 \%)$ & $2(100 \%)$ & $0(0 \%)$ & $0(0 \%)$ & $2(100 \%)$ & $0(0 \%)$ \\
\hline \begin{tabular}{l} 
E. coli \\
\hline $\begin{array}{l}\text { Enterococcus } \\
\text { faecalis }\end{array}$
\end{tabular} & $0(0 \%)$ & $0(0 \%)$ & $0(0 \%)$ & $0(0 \%)$ & $2(100 \%)$ & $0(0 \%)$ & $0(0 \%)$ & $2(100 \%)$ & $0(0 \%)$ \\
\hline $\begin{array}{l}\text { Klebsiella } \\
\text { pneumoniae }\end{array}$ & $6(60 \%)$ & $2(20 \%)$ & $0(0 \%)$ & $2(20 \%)$ & $1(10 \%)$ & $1(10 \%)$ & $3(30 \%)$ & $6(60 \%)$ & $1(10 \%)$ \\
\hline
\end{tabular}

\section{DISCUSSION}

In this study, to compare the antibiotic sensitivity pattern of different uropathogens, efficacy and cost effectiveness of different antibiotics used in the treatment of urinary tract infection in paediatric patients, we have included 100 patients less than 13 years of age admitted in paediatric ward/PICU at the tertiary healthcare center. The mean age of the study group was 5.89 years. Out of the 100 patients urine culture of $55 \%$ (55) patients were culture positive quite similar to study by Mashouf et al, which included 912 children in their study, carried out at a hospital in Iran out of which $34.2 \%$ were culture positive. ${ }^{16}$ In study carried out by Badhan $\mathrm{R}$ et al, in north India on 800 children, $26.7 \%$ (192) patients were found to be culture positive, this study differs from this study, might be because the study was carried on OPD patients with less severe infection. ${ }^{8}$

Out of the 55 culture positive patients in this study majority of the patients i.e. $50.90 \%$ ( 28 patients) were females and $49.10 \%$ (27 patients) were males, in study carried out by Badhan R et al, $54.2 \%$ of culture positive cases were seen in female patients. ${ }^{8}$ In a study conducted by Akram et al, in North India, maximum cases of culture positive cases of UTI were seen in females than in males. ${ }^{17}$ Findings of other studies conducted in different countries also suggested that culture positive UTI cases were seen more in females. ${ }^{18,19}$ Due to shorter length of urethra in females generally UTI are seen more in females. ${ }^{4}$ However, in a similar prospective study by Kalantar et al, on 1696 children of urinary tract infection it was found that culture positive cases were seen more in males than females in a ratio of $1.07: 1 .^{20}$ In a study by Taneja et al, in children uptill 12 years of age, UTI were seen more commonly in females. ${ }^{21}$

In this study, all 100\% (100) patients presented with fever, along with fever majority of the children presented with abdominal complaints followed by fever with urinary complaints together. In a study carried by Sharma et al, majority of the patients presented with fever and abdominal complaints. ${ }^{22}$ In one study by Brkic et al, fever was the most common presenting symptom in patients of UTI, seen in $54.9 \%$ of patients, this study is similar to this study in this context. ${ }^{19}$

In this study, the mean temperature of 100 children on arrival at paediatric OPD was 39.07 degree Celsius, in a study by Shaw et al, on febrile infants, $16 \%$ of febrile infant presented with temperature of $\geq 39$ degree Celsius at emergency department which is similar to this study. ${ }^{23}$ Bacteremia especially due to gram negative organisms like E. coli, Pseudomonas and Klebsiella pneumoniae are one of the most common causes of fever in children in developing countries. ${ }^{24}$

In this study, E. coli was the most common organism responsible for urinary tract infection accounting for $69.07 \%$ of all culture positive cases at the tertiary health care center ( $z$ value $>1.96$, significant on chi square), this is in compliance with studies in Iran by Kalantar et al, (54.8\%), Gupta et al, (64\%), Mashouf et al, (57.4\%). ${ }^{16,20,25}$ The above studies suggests that Ecoli is the most commonly isolated microorganism from patients of urinary tract infection from different locations. Klebsiella pneuminae is the second most common isolated organism in this setting from patients of UTI seen in $18.18 \%$ of all culture positive cases which is similar to studies such as Badhan R et al, (11.5\%), and also similar to studies by Taneja et al, (14.5\%) and Kumurya A et al (14\%). ${ }^{8,18,21}$ In this study Pseudomonas aeruginosa was isolated from $1.84 \%$ of patients and Acinetobacter was isolated from $3.64 \%$ of patients, low occurrence of these organisms is supported by Akram et al, study where no growth of these organisms were found. ${ }^{17}$ In Abdulhadi SK et al, study pseudomonas growth was seen in $2 \%$ of patients. ${ }^{18}$ However in one study by Taneja et al, pseudomonas aeruginosa was isolated from $10.9 \%$ of patients and Acinetobacter spp was isolated from $6.6 \%$ of patients, high occurrence of these microorganism in this study might be due to study was undertaken in patients admitted to PICU and increase number of indwelling catheters in situ led to increased incidence of these microorganisms in culture. ${ }^{21}$

In this study, Staphylococcus aureus and Enterococcus faecalis are seen in $3.64 \%$ and $3.64 \%$ of all culture positive cases of urinary tract infections respectively, Gram positive organisms are being considered one of the important causes of UTI, Staphylococcus aureus and Enterococcus faecalis have been isolated in very small numbers in various studies, still they are regarded as important cause of urinary tract infection. ${ }^{16,20,26}$

Present study depicted high sensitivity of Gram negative organism (E. coli, Klebsiella pnemoniae and Acinetobacter) to nitrofurantoin $(73.68 \%, 100 \%$ and $60 \%$ respectively) however pseudomonas was not found to be 
sensitive to nitrofurantoin, these gram negative uropathogens have also shown considerable efficacy towards cefixime, ceftriaxone and amikacin, these findings are similar to study by Badhan R et al. ${ }^{8}$ Similar results were seen in study by Biswas et al. ${ }^{27}$ Pseudomonas on the other hand had shown $100 \%$ sensitivity to ceftriaxone, similar results were obtained in study by Sharmin et al, which was collected from 60 patients of UTI in Dhaka. Gram negative uropathogens (E. coli and Klebsiella pnemoniae) have shown considerable sensitivity to nitrofurantoin, ceftriaxone, amikacin and imipenem, whereas Pseudomonas had shown $50 \%$ sensitivity to ciprofloxacin, however in study by Sharmin et al, ceftazidime has shown high sensitivity towards gram negative organism (Klebsiella pneumoniae and E. coli) which contrasts with our study where ceftazidime has shown high resistance pattern $(\approx 90 \%){ }^{28}$

In this study, gram positive uropathogens i.e. staphylococcus aureus and enterococcus faecalis were isolated from urine specimen of $3.64 \%$ of culture positive UTI patients, all cases of staphylococcus aureus were found to be sensitive to cotrimoxazole $(100 \%)$ whereas all samples of enterococcus faecalis were sensitive to nitrofurantoin $(100 \%)$, however in a study by Rezaee et al, Gram positive organisms were highly sensitive to amikacin and nitrofurantoin. ${ }^{2}$ In this study, uropathogens has shown highest resistance to ceftazidime $(43.64 \%)$ followed by cotrimoxazole $(34.55 \%)$, uropathogens were least resistant to ceftriaxone $(1 \%)$ followed by amikacin, nitrofurantoin and cefixime.

To choose a cost-effective antibiotic for community acquired urinary tract infections is always a challenging goal. Fever is one of the most common symptom of urinary tract infections in children, it is a marker of bacteraemia and renal parenchymal involvement. To compare the efficacy of different antibiotics used in children suffering from UTI author have considered time required for defervescence and degree of defervescence/hour (>37.0degree celsius of axillary temperature was considered fever). While considering time required for defervescence authors didnt found any significant difference between efficacy of different antibiotics like amikacin (33.7 hours), ceftriaxone (31.09 hours), cefixime (33.87 hours), coamoxyclav (38 hours), cefopodoxime (37.33 hours), imipenem (32 hours), cotrimoxazole (33.11 hours), ciprofloxacin (37.11 hours) and cefotaxime (30 hours) (pvalue $>0.05$ ), however there was a significant difference in efficacy in terms of time required for defervescence in between above antibiotics and nitrofurantoin ( 53.70 hours) (p-value $<0.05$ ), nitrofurantoin is not much effective in febrile state since its inhibitory concentration in blood is not attained. ${ }^{29}$

When authors compared efficacy of antibiotics in terms of degree of defervescenc /hour, Inj. Ceftriaxone (0.07-degree celsius/hour) was found to be more efficacious than syp/tab coamoxyclav (0.05 degree Celsius/hour) and tab ciprofloxacin $(0.05$ degree Celsius/hour) (p-value <0.05).
However, there was no significant statistical difference between Inj. ceftriaxone and other antibiotics i.e. cefixime (0.06 degree Celsius /hour), cotrimoxazole (0.065 degree Celsius/hour), Inj. Amikacin (0.063 degree Celsius/hour) and cefopodoxime (0.055 degree/Celsius) ( $p$-value $>0.05)$. However, there was significant difference between tab nitrofurantoin (0.036/hour) and other drugs in terms of defevescence/hour, ( $p$-value <0.05), Inj. cefotaxime and Inj. imipenem was not taken into consideration since only only one patient received this medication respectively.

In a study by Chang et al, in patients suffering from uncomplicated pyelonephritis, no statistical difference in efficacy was found between cefuroxime and cefotaxime (while considering time required for fever to come down), in one non inferiority clinical trial comparing efficacy between Tab coamoxyclav and Inj ceftriaxone in children suffering from UTI, time required for fever to come down to normal level was considered as a measure of efficacy and secondary outcome, no statistical difference in efficacy was found in this study between Tab coamoxyclav and Inj ceftriaxone, though other parameters like renal scarring and microbiological urine analysis were also considered as a measure of efficacy in this study still there was no statistical difference was found, however in this study Inj. ceftriaxone was found to be more efficacious than tab coamoxyclav ( $\mathrm{p}$-value $<0.05$ on multiple comparison test) which is in contrast with this study. ${ }^{30,31}$

Cost effective analysis is cost per unit health gained, cost effective analysis has a huge application in developing countries where resources are limited and health problems are more prevalent, authors developed a model for our study based on the principles of cost effective analysis to compare cost effectiveness between different antibiotics used in children suffering from urinary tract infections, authors measured cost effective analysis as cost of antibiotic required for fever to come down/degree through which fever came down. ${ }^{12}$ In this study cotrimoxazole, ciprofloxacin and nitrofurantoin were found to be the most cost effective drugs with CER of 2.8,8.35 and 11.8 respectively, there was statistical significant difference in CER of cotrimoxazole, ciprofloxacin, nitrofurantoin and other drugs (cefixime, cefpodoxime, ceftriaxone, amikacin, coamoxyclav) ( $\mathrm{p}$-value $<0.05$ ) however there was no statistical difference in CER in between cotrimoxazole, ciprofloxacin and nitrofurantoin ( $\mathrm{p}$-value $>0.05)$.

Cefixime and cefpodoxime were found to be more cost effective than other drugs with CER of 9.97 and 11.93 respectively ( $\mathrm{p}$-value $<0.05$ on multiple comparison test) however there was no statistically significant difference between cefixime and cefpodoxime with respect to CER ( $\mathrm{p}$-value $>0.05$ on multiple comparisons). In a randomized controlled trial by Bosmans et al, in comparing cost effectiveness between cranberry capsules and cotrimoxazole for prophylaxis of UTI in premenopausal women, cotrimoxazole was found to be more cost effective, in this study cotrimoxazole was found to be more 
cost effective however our study was done to compare antibiotics for treatment of UTI in children and study by Bosmans et al, was done to know prophylactic CER in premenopausal women. ${ }^{32}$

\section{CONCLUSION}

In this current study, authors found $E$. coli is the most common uropathogen isolated. Ceftriaxone, cefixime, cotrimoxazole and amikacin were found to be most efficacious. Cotrimoxazole, ciprofloxacin and nitrofurantoin were found to be most cost-effective antimicrobial.

Funding: No funding sources Conflict of interest: None declared

Ethical approval: The study was approved by the Institutional ethics committee (No: DMIMS(DU)/IEC/2016-17/4060)

\section{REFERENCES}

1. Urinary Tract Infection: Symptoms, Diagnosis, and Treatment. Healthline, 2015. Available at: https://www.healthline.com/health/urinary-tractinfection-adults. Accssed at 8 Sep 2018.

2. Rezaee MA, Abdinia B. Etiology and antimicrobial susceptibility pattern of pathogenic bacteria in children subjected to UTI. Medicine (Baltimore), 2015. Available at: https://www.ncbi.nlm.nih.gov/pmc/articles/PMC4616 821\%. Accessed 7 Sep 2018.

3. Freedman AL. Urologic diseases in North America project: trends in resource utilization for urinary tract infections in children. J Urol. 2005;173(3):949-54.

4. Montini G, Tullus K, Hewitt I. Febrile urinary tract infections in children. $\mathrm{N}$ Engl $\mathrm{J}$ Med. 2011;365(3):239-50.

5. The diagnosis, evaluation and treatment of acute and recurrent pediatric urinary tract infections. Available at:

https://www.ncbi.nlm.nih.gov/pmc/articles/PMC4652 790/. Accessed 31 Aug 2018.

6. Kaushik V, Chaudhary SR. Study for prevalence of urinary tract infection (UTI) in febrile children and to assess the validity of microscopic urine analysis in the diagnosis of UTI. Urin Tract Infect. 2017;4(4):4.

7. Yakubov R, Akker M, Machamad K, Hochberg A, Nadir E, Klein A. Antimicrobial resistance among uropathogens that cause childhood communityacquired urinary tract infections in Central Israel. Pediatr Infect Dis J. 2017;36(1):113-5.

8. Badhan R, Singh DV, Badhan LR, Kaur A. Evaluation of bacteriological profile and antibiotic sensitivity patterns in children with urinary tract infection: a prospective study from a tertiary care center. Ind $\mathrm{J}$ Urol. Available

at: http://www.indianjurol.com/article.asp?issn=09701591; year $=2016$; volume $=32 ;$ issue $=1$; spage $=50$; epage =56; aulast=Badhan. Accessed 31 Aug 2018 .
9. Bader MS, Loeb M, Brooks AA. An update on the management of urinary tract infections in the era of antimicrobial resistance. Postgrad Med. 2017;129(2):242-58.

10. Stein R, Dogan HS, Hoebeke P, Kočvara R, Nijman RJM, Radmayr C, et al. Urinary tract infections in children: EAU/ESPU guidelines. Eur Urol. 2015;67(3):546-58.

11. Epidemiology of urinary tract infections: incidence, morbidity, and economic costs. Available at: ://www.sciencedirect.com/shttpscience/article/pii/S00 02934302010549. Accessed 31 Aug 2018.

12. Estill J, Salazar-Vizcaya L, Blaser N, Egger M, Keiser $\mathrm{O}$. The cost-effectiveness of monitoring strategies for antiretroviral therapy of HIV infected patients in resource-limited settings: software tool. PloS One. 2015;10(3):e0119299.

13. Fenwick EAL, Briggs AH, Hawke CI. Management of urinary tract infection in general practice: a costeffectiveness analysis. Br J Gen Pract. 2000;5.

14. Vallejo-Torres L, Pujol M, Shaw E, Wiegand I, Vigo JM, Stoddart M, et al. Cost of hospitalized patients due to complicated urinary tract infections: a retrospective observational study in countries with high prevalence of multidrug-resistant Gram-negative bacteria: the combacte-magnet, rescuing study. BMJ Open. 2018;8(4):e020251.

15. Linhares I, Raposo $\mathrm{T}$, Rodrigues A, Almeida A. Frequency and antimicrobial resistance patterns of bacteria implicated in community urinary tract infections: a ten-year surveillance study (2000-2009). BMC Infect Dis. 2013;13:19.

16. Mashouf RY, Babalhavaeji H, Yousef J. Urinary tract infections: bacteriology and antibiotic resistance patterns. Ind Pediatr. 2009;46(7):617-20.

17. Akram M, Shahid M, Khan AU. Etiology and antibiotic resistance patterns of community-acquired urinary tract infections in JNMC Hospital Aligarh, India. Ann Clin Microbiol Antimicrob. 2007;6:4.

18. Kumurya A, Yashua AH, Uba A. Organisms causing urinary tract infection in pediatric patients at Murtala Mohammed Specialist Hospital, Kano, Nigeria. Int $\mathbf{J}$ Biomed Health Sci. 2008;4:165-7.

19. Brkic S, Mustafic S, Nuhbegovic S, Ljuca F, Gavran L. Clinical and epidemiology characteristics of urinary tract infections in childhood. Med Arh. 2010;64(3):135-8.

20. Kalantar E, Motlagh $M$ esmaeel, Lornejad H, Reshadmanesh N. Prevalence of urinary tract pathogens and antimicrobial susceptibility patterns in children at hospitals in Iran. Arch Clin Infect Dis. 2008;3(3). Accessed 5 September 2018. Available at: http://journals.sbmu.ac.ir/infectiousinvisible/article/vi ew/209.

21. Taneja N, Chatterjee SS, Singh M, Singh S, Sharma M. Pediatric urinary tract infections in a tertiary care center from north India. Indian J Med Res. 2010;5.

22. Sharma A, Shrestha S, Upadhyay S, Rijal P. Clinical and bacteriological profile of urinary tract infection in 
children at Nepal Medical College Teaching Hospital. Nepal Med Coll J NMCJ. 2011;13(1):24-6.

23. Shaw KN, Gorelick M, McGowan KL, Yakscoe NM, Schwartz JS. Prevalence of urinary tract infection in febrile young children in the emergency department. Pediatrics. 1998;102(2):e16-e16.

24. Christopher A, Mshana SE, Kidenya BR, Hokororo A, Morona D. Bacteremia and resistant gram-negative pathogens among under-fives in Tanzania. Ital $\mathbf{J}$ Pediatr. 2013;39:27.

25. VG. Antibiotic resistance pattern in uropathogens. Ind J Med Microbiol. 2002;20(2):96.

26. Habte TM, Dube S, Ismail N, Hoosen AA. Hospital and community isolates of uropathogens at a tertiary hospital in South Africa. South Afr Med J Suid-Afr. 2009;99(8):584-7.

27. Biswas D, Gupta P, Prasad R, Singh V, Arya M, Kumar A. Choice of antibiotic for empirical therapy of acute cystitis in a setting of high antimicrobial resistance. Ind J Med Sci. 2006;60(2):53-8.

28. Sharmin S, Alamgir F, Fahmida M, Saleh AA. Antimicrobial sensitivity pattern of uropathogens in children. Bangladesh J Med Microbiol. 2010;3(2):18.

29. Prais D, Straussberg R, Avitzur Y, Nussinovitch M, Harel L, Amir J. Bacterial susceptibility to oral antibiotics in community acquired urinary tract infection. Arch Dis Child. 2003;88(3):215-8.

30. Chang U-I, Kim HW, Wie S-H. Comparison of second- and third-generation cephalosporin as initial therapy for women with community-onset uncomplicated acute pyelonephritis. Yonsei Med J. 2015;56(5):1266-73.

31. Montini G, Toffolo A, Zucchetta P, Dall'Amico R, Gobber D, Calderan A, et al. Antibiotic treatment for pyelonephritis in children: multicentre randomised controlled non-inferiority trial. BMJ. 2007;335(7616):386.

32. Bosmans JE, Beerepoot MAJ, Prins JM, Riet G, Geerlings SE. Cost-Effectiveness of cranberries vs antibiotics to prevent urinary tract infections in premenopausal women: a randomized clinical trial. PLoS One. 2014;9(4).

Cite this article as: Yadav NS, Pathak SS.

Management of urinary tract infections in children: antimicrobial sensitivity pattern, efficacy and pharmacoeconomics. Int J Basic Clin Pharmacol 2019;8:1361-70. 